\title{
130. Chromosomal Polymorphisms in the Biwa Trout (Salmonidae) with an Increase or Decrease in Constitutive Heterochromatin
}

\author{
By Takayoshi UedA, ${ }^{* 1}$ Yoshio OJIma,**) and Kenji NAKA***) \\ (Communicated by Sajiro Makıno, M. J. A., Dec. 12, 1985)
}

Ueda and Ojima (1983a, b) on the basis of C-banding karyotype analyses suggested that an increase or decrease in constitutive heterochromatin may have cccurred in closely relation to the karyotype differentiation in the Salmonidae. Yosida (1979) emphasized the close relation between the differentiation of the nucleolus organizer regions (NORs) and species evolution in Rattus species. Although several reports on the N-banding karyotypes in fishes have been published (Kligerman and Bloom 1977; Howell and Black 1979; Kornfield et al. 1979; Ojima and Yamano 1980; Foresti et al. 1981; Uwa and Ojima 1981; Uwa and Iwata 1981; Uwa et al. 1981, 1982, 1983; Gold 1984; Kitayama and Ojima 1984; Takai and Ojima 1984), there have been none on the Salmonidae.

In the present report, $\mathrm{C}$ - and $\mathrm{N}$-banding karyotypes as well as routine-Giemsa karyotypes of the Biwa trout (Oncorhynchus masou) are described, and chromosomal polymorphisms with regard to an increase or decrease in constitutive heterochromatin are discussed.

Materials and methods. Two females and 4 males of the Biwa trout $(O$. masou) collected from the Lake Biwa were used. The fins were cultured to obtain dividing cells efficiently. Chromosome slides were made by the culture method (Ueda et al. 1984) and the routine air-drying method with conventional Giemsa stain. The C- (Sumner 1972) and N-banding techniques (Howell and Black 1980) were also used for detailed chromosome analyses. The nomenclature of the chromosomes followed the Levan et al. system (1964).

Results. Fig. 1 shows a routine-Giemsa karyotype (upper rows) and the C-banding karyotype (lower rows). The chromosome number is $66(2 n)$ and the chromosome constitution is 38 meta- (M) and submetacentrics (SM), 26 subtelo(ST) and acrocentrics (A), and $2 \mathrm{SM}$ or ST. Therefore, the NF (fundamental number) is 104-106. This karyotypic form is characterized by 2 SM or ST with a satellite-like structure and intense C-bands on the short arms (underlined in Fig. 1). Two forms-large and small-exist and each individual can be divided into 3 groups on the basis of whether it carries 2 large, 2 small, or one large and one small structure (Fig. 3). The part of this structure had silver stained NORs (Ag-NORs) (Figs. 2 and 3).

Discussion. The chromosomal polymorphisms in the Biwa trout are accompanied by an increase or decrease in constitutive heterochromatin. The size of the Ag-NORs has been regarded as a main cause for the variety of the marker chromosomes. Generally speaking, rDNA content and silver staining intensity are closely correlated (Warburton and Henderson 1979). If that is the case, the

*) Dept. of Biol., Fac. of Ed., Utsunomiya Univ., Utsunomiya 321, Japan.

**) Dept. of Biol., Fac. of Sci., Kwansei Gakuin Univ., Nishinomiya 662, Japan.

***) Shiga Pref. Fish. Exp. Sta., Hikone 522, Japan. 


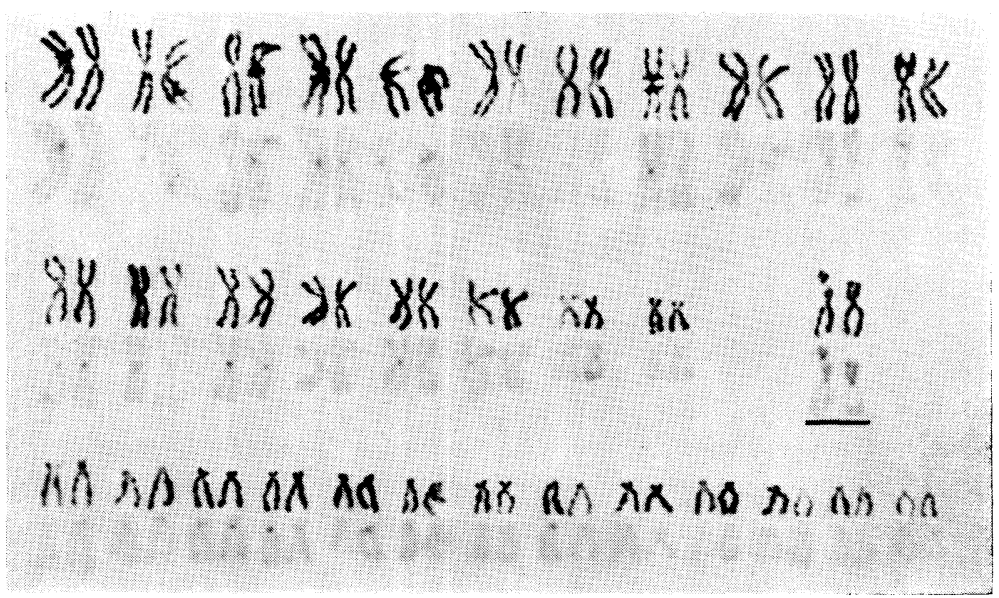

Fig. 1. Karyotype of the Biwa trout (O. masou) ; conventional (upper rows) and C-banding stain (lower rows). The underline indicates two chromosomes with the satellite-like structure and intense C-bands on the short arms.

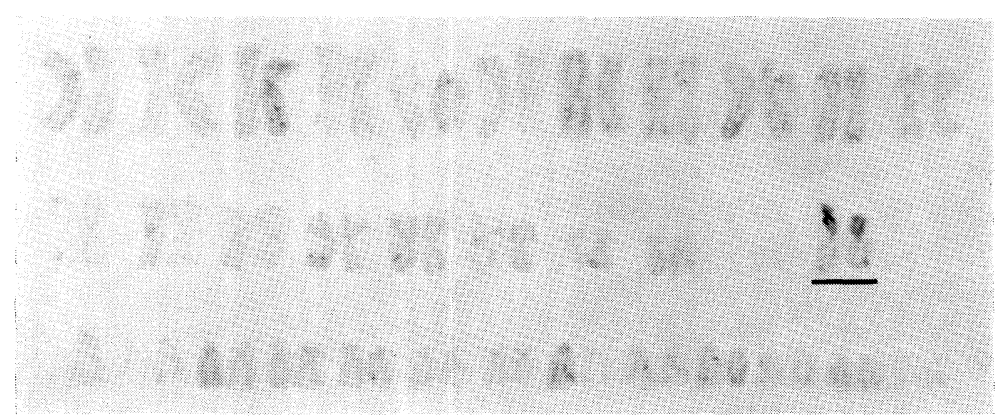

Fig. 2. N-banding karyotype of the Biwa trout (O. mascu). The underline indicates two chromosomes with the satellite-like structure and intense N-bands near the structure regions.

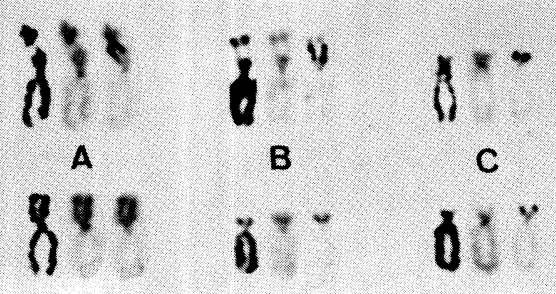

Fig. 3. Chromosomes with the satellite-like structure of three individuals (A, B and C) in the Biwa trout (O. masou); conventional (left), C-banding (middle) and $\mathrm{N}$-banding stain (right).

rDNA content of the Biwa trout are diverse in every individual.

The chromosomal polymorphisms in $O$. masou occur in both sexes. Very similar polymorphisms were observed in O. nerka and Salvelinus malma, and these were also found in both sexes (Ueda and Ojima 1983b, 1984a). On the other hand, similar marker chromosomes observed in the lake trout (Salvelinus namaycush) were considered to be sex chromosomes because of their size differ- 
ence in each sex (Phillips and Ihssen 1985). The two kinds of marker chromosomes, namely those in the lake trout and those reported here in the Biwa trout, appear to be completely different from each other. The chromosomal polymorphisms accompanied by an increase or decrease in constitutive heterochromatin, which we observed, may be the beginning of sex chromosome differentiation. But sex chromosome differentiation in O. nerka and Salmo gairdneri by means of Robertsonian translocation or chromosomal change from ST to A was recognized by Thorgaard $(1977,1978)$, Ueda and Ojima (1984a, b). More studies throughout the Salmonidae will be required to clarify these problems.

Acknowledgment. We are very indebted to Dr. Sajiro Makino, M. J. A., Professor Emeritus, Hokkaido University, for improvement of the manuscript and revision of data.

\section{References}

Foresti, F. et al. (1981): Cytogenet. Cell Genet., 31, 137-144.

Gold, J. R. (1984) : Copeia, 1984, 133-139.

Howell, W. M., and Black, D. A. (1979) : ibid., 1979, 544-546.

- (1980): Experientia, 36, 1014-1015.

Kitayama, E., and Ojima, Y. (1984) : Proc. Japan Acad., 60B, 58-61.

Kligerman, A. D., and Bloom, S. E. (1977): Cytogenet. Cell Genet., 18, 182-196.

Kornifield, J. L. et al. (1979) : Evolution, 33, 1-14.

Levan, A. et al. (1964): Hereditas, 52, 201-220.

Ojima, Y., and Yamano, T. (1980) : Proc. Japan Acad., 56B, 551-556.

Phillips, R. B., and Ihssen, P. E. (1985) : Cytogenet. Cell Genet., 39, 14-18.

Sumner, A. T. (1972): Exp. Cell Res., 75, 304-306.

Takai, A., and Ojima, Y. (1984): Proc. Japan Acad., 60B, 410-413.

Thorgaard, G. H. (1977) : Science, 196, 900-902. (1978) : Can. J. Genet. Cytol., 20, 349-354.

Ueda, T., and Ojima, Y. (1983a) : Proc. Japan Acad., 59B, 259-262.

-_ (1983b) : ibid., 59B, 343-346.

Ueda, T. et al. (1984) : Bull. Japan. Soc. Sci. Fish., 50, 1331-1336.

Ueda, T., and Ojima, Y. (1984a) : ibid., 50, 1495-1498. (1984b) : ibid., 50, 1499-1504.

Uwa, H., and Ojima, Y. (1981): Proc. Japan Acad., 57B, 39-43.

Uwa, H., and Iwata, A. (1981) : CIS, 31, 24-26.

Uwa, H. et al. (1981) : Proc. Japan Acad., 57B, 95-99.

Uwa, H. et al. (1982): CIS, 33, 15-17.

Uwa, H. et al. (1983) : Proc. Japan Acad., 59B, 43-47.

Warburton, D., and Henderson, A. S. (1979) : Cytogenet. Cell Genet., 24, 168-175.

Yosida, T. H. (1979) : Proc. Japan Acad., 55B, 481-486. 